Cinémas

Revue d'études cinématographiques

Journal of Film Studies

\title{
Scénarios du vécu : cinéma, histoire et récit de vie
}

\section{Marie-Claude Taranger}

Volume 9, numéro 2-3, printemps 1999

Les Scénarios fictifs

URI : https://id.erudit.org/iderudit/024790ar

DOI : https://doi.org/10.7202/024790ar

Aller au sommaire du numéro

Éditeur(s)

Cinémas

ISSN

1181-6945 (imprimé)

1705-6500 (numérique)

Découvrir la revue

Citer cet article

Taranger, M.-C. (1999). Scénarios du vécu : cinéma, histoire et récit de vie. Cinémas, 9(2-3), 123-145. https://doi.org/10.7202/024790ar

\section{Résumé de l'article}

Ce texte explore les relations entre récits de vie et scénarios, à partir d'un corpus d'entretiens portant sur les années 1914-1945. Il part d'un constat a priori surprenant : ces récits de vie ont beaucoup à voir avec des scénarios de films, ou plus largement des scénarios médiatiques, auxquels ils empruntent toutes sortes d'éléments. On montre d'abord comment les récits de vie se réapproprient ces matériaux rapportés, qu'ils intègrent très précisément dans leurs cohérences spécifiques. On explore ensuite les fonctions de ce recours à des modèles consacrés. L'étude aboutit à mettre en question la distinction entre vécu et fiction, parole et cinéma, et à préciser les relations entre récit personnel et imaginaire collectif. 


\title{
Scénarios du vécu: cinéma, histoire et récit de vie
}

\section{Marie-Claude Taranger}

\begin{abstract}
RÉSUME
Ce texte explore les relations entre récits de vie et scénarios, à partir d'un corpus d'entretiens portant sur les années 1914-1945. Il part d'un constat a priori surprenant: ces récits de vie ont beaucoup à voir avec des scénarios de films, ou plus largement des scénarios médiatiques, auxquels ils empruntent toutes sortes d'éléments. On montre d'abord comment les récits de vie se réapproprient ces matériaux rapportés, quïls intègrent très précisément dans leurs cohérences spécifiques. On explore ensuite les fonctions de ce recours à des modèles consacrés. Létude aboutit à mettre en question la distinction entre vécu et fiction, parole et cinéma, et à préciser les relations entre récit personnel et imaginaire collectif.
\end{abstract}

\section{ABSTRACT}

This text explores the relations between real-life narratives, based on a body of interviews led between 1914 and 1945, and screenplays. Its point of departure may at first appear surprising: these real-life narratives have much in common with film screenplays, or more generally with mass-media scripts from which they borrow all types of elements. We will first see how reallife narratives reappropriate these exterior materials and integrate them very precisely within their own coherent scheme. We will then examine the functions of this recourse to established models. The study concludes by calling into question the distinction between actual experience and fiction, speech and cinema, and by specifying the relations between personal narrative and collective imagination. 
Tout commence avec une étrange rencontre.

Interviewée dans le cadre d'une recherche historique, une femme témoigne de son expérience personnelle et raconte sa vie: précisément, un épisode situé pendant la Deuxième Guerre mondiale en France, l'exode de juin 1940. Elle était de ces populations du Nord du pays que l'arrivée des Allemands avait jetées sur les routes avec familles et bagages. Elle raconte donc: la fuite avec sa mère, les heures et les jours de marche, les bombardements de l'aviation ennemie. Puis, tout d'un coup, on s'aperçoit qu'elle ne raconte plus son histoire à elle, mais un film. Elle le nomme: Jeux interdits (René Clément, 1951). La première séquence du film présente la même situation, mais bien sûr avec d'autres personnages: Brigitte Fossey (la petite héroïne), ses parents, son chien, la foule qui les entoure. Et ce sont eux qu'évoque maintenant le récit de notre narratrice: "Ses parents la couvrent $[. .$.$] . On voit tous les gens qui défilent [\ldots]$. En dessus, il y a un mitraillage par les avions allemands" (entretien $\left.\mathrm{n}^{\circ} 378\right)^{\prime}$.

L'irruption de ce scénario de film dans cette histoire de vie conduit bientôt à un étrange imbroglio. Car en racontant Jeux interdits, la narratrice ne cesse pas de raconter son histoire propre. En fait, elle alterne, entrelace et finit par fondre complètement les deux scénarios. Les bombardements du film et les bombardements qu'elle a vécus ne constituent plus qu'un seul événement, auquel elle participe aussi bien que les personnages de l'écran, jusqu'à devenir elle-même héroïne du film: au paroxysme de l'interpénétration des deux récits, les personnes finissent par être équivalentes, la première et la troisième, je (nous) et elle (ils) :

Il y a un mitraillage par les avions allemands. Ils essayaient de nous empêcher de continuer. Eh bien, ils se plaquent dessus. C'est les parents qui sont tués. C'est comme ça qu'elle s'en va dans cette ferme avec le petit. Et c'était vrai. Moi je sais que je l'ai vécu et c'est vrai, puisque j'ai été... On m'a appelée après madame Goudron, parce que je suis tombée dans le goudron (entretien n"378). 
La première fois que j'ai écouté l'enregistrement de cet entretien, je me suis perdue dans ce récit : que racontait-elle donc? sa vie ou un film? Mais mon désarroi était lié à un souci de cohérence superficielle (et grammaticale) déplacé en la circonstance: de fait, dans la fusion des situations et des personnages, le mouvement de la narration ne cessait jamais d'être saisissable. Son étrangeté apparente a cependant suscité chez moi un étonnement qui s'est bientôt mué en questionnement et m'a conduite à explorer plus avant ce phénomène de contamination. Je me propose d'exposer ici les résultats de cette investigation.

\section{Cadre et méthode}

Le cadre de cette recherche est une enquête orale qui porte sur les années 1914-1945. Menée pendant plus de 10 ans dans le Midi de la France par une équipe de chercheurs et d'étudiants, elle a permis de recueillir de véritables récits de vie, où des témoins directs racontent ce qu'a été leur histoire durant cette période. Nous avons étudié ailleurs dans une perspective globale l'apport de ces témoignages à l'Histoire ${ }^{2}$. La question qui nous intéresse ici participe plus spécifiquement d'une analyse des stratégies narratives et de la construction de la mémoire. Comment s'élaborent ces scénarios du vécu? et comment s'articulent-ils aux autres scénarios existants, particulièrement à ceux qu’offre le cinéma ${ }^{3}$ ? On verra qu'une telle étude, comme le suggérait déjà l'exemple de Jeux interdits, peut aboutir à mettre en question un partage des genres a priori qui permettrait de distinguer d'un côté le cinéma, la fiction, le scénario, et de l'autre, le témoignage, l'histoire, le document. Loin de l'évidence de ces oppositions de bon sens, et suivant la logique même qui conduit à s'intéresser aux «scénarios fictifs", la prise en compte d'un matériau d'autant plus spécifique qu'il s'agit de récit "oral" peut permettre de replacer le fonctionnement du scénario dans la complexité effective qui caractérise la circulation sociale des récits.

Il serait ici hors de propos d'exposer en détail les procédures utilisées dans notre enquête, mais il est néanmoins nécessaire de préciser deux points importants concernant l'élaboration des récits de vie étudiés. En premier lieu, les témoins - pour la 


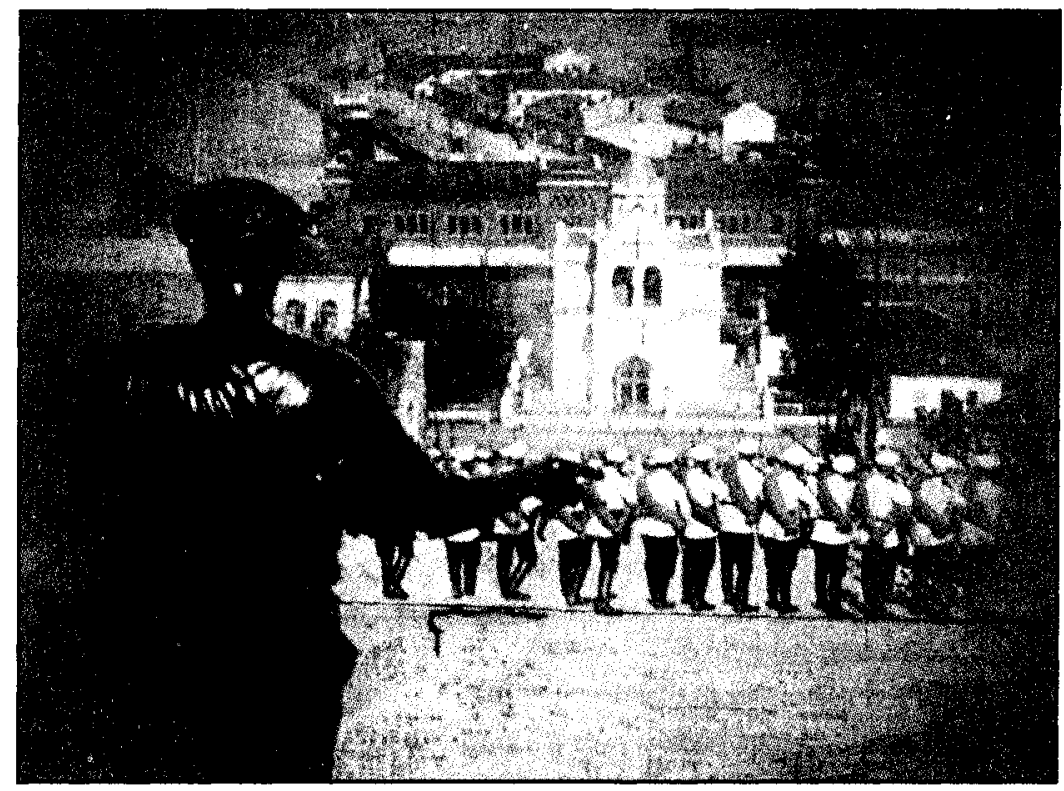

\section{Le Cuirassé Potemkine d'Eisenstein (1925)}

plupart des témoins ordinaires de l'histoire - étaient invités à raconter leur "histoire personnelle", ce qu'ils avaient euxmêmes vécu, vu, observé. Les entretiens étaient d'autre part des entretiens non directifs: le rôle des interviewers se limitait à susciter la parole - et le cas échéant à la relancer — en s'efforçant de laisser aux narrateurs le maximum d'autonomie dans la construction de leur récit. De fait, et malgré quelques exceptions, les témoins ont manifestement pu développer leurs stratégies narratives propres.

\section{Emprunts}

Ces faits rendent d'autant plus frappant le constat qu'impose l'ensemble du matériau recueilli, constat qui rejoint l'exemple de Jeux interdits: bien que les narrateurs évoquent surtout leur vécu, leurs récits sont truffés d'éléments rapportés. Y foisonnent des références ou des emprunts au Cuirassé Potemkine (Eisenstein, 1925) et au Dernier Métro (Truffaut, 1980), aux actualités Paramount et aux débats télévisés, à des émissions de radio, à des livres d'histoire et à bien d'autres sources encore. 


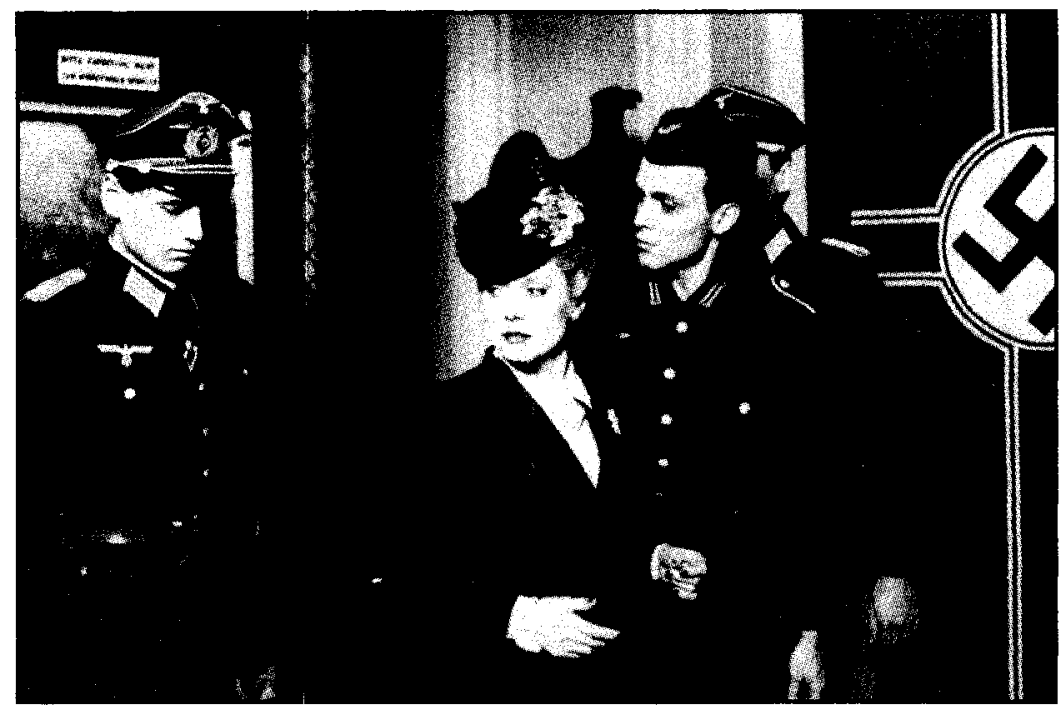

\section{Le Dernier Métro de François Truffaut (1980)}

Ainsi, entre l'expérience directe et le récit qui en est fait s'interposent d'autres récits et d'autres paroles: fournis par l'ensemble des médias auxquels ont eu accès les témoins, ils se mêlent à l'histoire directement vécue et deviennent la matière grâce à laquelle s'élaborent les "témoignages".

Mais peut-on continuer alors à parler de témoignage? Pour le chercheur, ces matériaux rapportés sont à certains égards source de désillusion. Du récit de vie, du témoin ordinaire, on attend qu'ils fournissent une expérience authentique, des faits, de la vérité, du vécu, bref de l'information brute. Or voici qu'il livre des données extérieures et postérieures, élaborées par d'autres que lui et qui renvoient à cette histoire médiatique et officielle dont l'histoire orale cherche à s'affranchir. Que faire? La première tentation est d'opérer un tri et de déblayer les éléments "étrangers" pour ne garder que le témoignage original, comme on cherche à retrouver un tableau sous des retouches ultérieures ou comme on essaie d'éliminer les interpolations d'un texte.

La tâche cependant apparaît vite dénuée de sens.

D'abord parce que le tri en lui-même est impraticable: comment parvenir à déterminer dans tous les cas l'origine de ce qui est raconté ? Certes les récits précisent souvent leurs 
références et ils donnent divers renseignements sur leurs sources. Mais beaucoup de références et d'emprunts sont implicites, et très souvent sans doute inconscients. On peut encore parvenir à en identifier quelques-uns, parce qu'on reconnaît ici une scène, là les mots d'une citation: mais cela manifestement est affaire de chance et d'intuition, et pour quelques emprunts repérés, de nombreux autres nécessairement échappent à l'analyse.

Là n'est pas pourtant l'essentiel. Car l'examen des cas où l'origine du matériau est repérable (quand emprunts et références sont explicites ou ostensibles) conduit à mettre en cause le bienfondé du tri. L'idée qu'il pourrait y avoir un récit authentique, qui serait "de surcroît " agrémenté ou parasité de références et d'emprunts, se révèle n'être qu'une illusion: loin que le témoignage direct et l'histoire personnelle aient des contours précis, l'imbrication est extrême aussi bien entre les matériaux de diverses origines qu'entre l'histoire d'un individu et d'autres histoires, celles d'un frère, d'un mari, d'un quartier, d'un village. Des éléments apparemment hétérogènes participent d'une même construction narrative et contribuent à mettre en place une cohérence générale dans laquelle la distinction entre souvenir personnel et mémoire empruntée perd toute pertinence. Au point qu'un fait qui paraît rapporté (et l'est effectivement) permet souvent de raconter sa vie tout autant que le fait qu'on a vécu soi-même. C'est cette imbrication que je voudrais maintenant m'attacher à décrire.

Parmi les matériaux empruntés, le cinéma occupe une place privilégiée par la fréquence et la diversité de ses interventions. Aussi sera-t-il pour notre description un outil privilégié. Il n'en sera pas pour autant l'outil exclusif: puisque le phénomène que nous nous proposons d'appréhender n'est pas limité au seul cinéma, il apparaît pertinent de replacer l'investigation spécifique relative au film dans le contexte plus large dont elle participe.

\section{Modes}

\section{- TRESSAGE}

Racontant son histoire, une ancienne couturière de la banlieue parisienne évoque l'entrée des Allemands à Paris en 1940 et la libération de la capitale en 1944 (entretien $n^{\circ}$ 303). Son 
récit nous apprend qu'elle a pris connaissance de façon différente de ces deux événements comparables: en 1940, elle a assisté avec son père au défilé des troupes hitlériennes, alors qu'en 1944, elle n'a vu la libération qu'au cinéma (dans les actualités). Le cinéma vient donc combler ici les lacunes de l'expérience directe. Mais il est clair que, dans le récit, l'origine des images importe moins que la symétrie des situations et la cohérence du scénario que le recours à l'information médiatique permet de construire.

Second exemple, avec lequel nous retrouvons non seulement la guerre mais l'exode. Une des narratrices, élève en 1940 d'un pensionnat marseillais (elle avait 10 ans), relate ainsi ses souvenirs:

Il y avait des enfants parmi nous qui étaient orphelins par la guerre / par l'exode des gens du Nord qui étaient descendus / quand on voit ces trucs-là / qui étaient descendus en tout portant sur des poussettes / sur le dos / quand il n'y avait plus d'essence / qui laissaient les voitures sur le bord des routes et qui filaient. Et nous avions beaucoup d'enfants de là (entretien $n^{\circ} 213$ ).

Ici encore, un récit unique se bâtit sur deux sources d'informations différentes: le cinéma pour l'événement lui-même ("quand on voit ces trucs-là») et l'expérience directe pour ses suites (les orphelins dans l'école). On ne peut qu'être frappé par le fait que les détails les plus concrets (les poussettes, les voitures au bord de la route) ne viennent pas de la vie, mais de l'écran.

Voici un autre exemple emprunté au registre des récits décrivant comment, pendant la guerre, les élégantes, faute de bas, se maquillaient les jambes :

On faisait un genre de peinture / je ne sais pas ce qu'on y mettait dedans / on y mettait une poudre / ça devait être comme une poudre de riz qu'on délayait dans de l'eau / on faisait un peu épais et on se le passait au pinceau tout le long des jambes (rire) / ec pour le faire bien bien régulier derrière / on s'allongeait sur la table / et il y avait toujours une copine qui le passait / comme ça / et avec un crayon noir on faisait la raie / et ça faisait les bas (entretien $n^{\circ} 213$ ). 
Malgré la précision de la description, la narratrice qui donne tous ces détails ne les a pourtant jamais vécus: "Ce n'était pas tout le monde qui pouvait se payer le produit pour faire ça. Moi, d'ailleurs, je ne l'ai jamais fait. Ma mère ne m'aurait pas donné l'argent pour acheter ça» (entretien n²13).

Elle s'est contentée de voir le résultat "de loin" sur les élégantes d'un autre monde.

En revanche, nombre de films ont montré des scènes de ce genre, et leur influence est d'autant plus probable ici que les allusions à cette pratique se sont multipliées comme par enchantement dans notre corpus après la sortie du Dernier Métro de François Truffaut qui justement évoque ces bas dessinés. Cette influence cependant ne s'exerce pas n'importe comment. D'une part, le récit se réapproprie manifestement la matière empruntée au point que son caractère rapporté ne peut guère apparaître qu'à l'analyse: l'exemple cité, dans sa vivacité et son pittoresque, le montre avec une netteté particulière. D'autre part, l'apport extérieur s'intègre dans une logique générale du scénario; les récits où se retrouve cette scène sont les récits de narratrices qui étaient à l'époque de jeunes ou de très jeunes filles et qui heurtaient leur désir de plaire aux difficultés de la vie quotidienne: les mêmes récits abondent en bigoudis intempestifs (les bombardements!) et en robes rétrécies ou déteintes.

Trois exemples, parmi des dizaines. Ils different par de nombreux traits: les modalités de l'emprunt (plus ou moins explicite), le type de faits empruntés (grands événements historiques ou détails de la vie quotidienne), la nature de la source (document ou fiction), et également le niveau d'articulation entre matériau vécu et matériau filmique, le cinéma pouvant apporter au récit un élément autonome (libération de Paris ou dessin des bas) ou une partie d'un ensemble (l'exode), ou superposer son image à celle du vécu (la scène de Jeux interdits). Mais ces exemples divers participent également d'un même phénomène et illustrent la façon dont les scénarios du vécu peuvent se construire en puisant dans les scénarios de l'écran: le cinéma vient ajouter sa matière à celle de l'expérience directe, mais cette matière est intégrée dans une élaboration propre dont la cohérence est en général très marquée. 


\section{- ÉCHANGES}

Bien évidemment, le fonctionnement ainsi décrit ne concerne pas le seul cinéma; les livres, les journaux, la radio peuvent jouer un rôle analogue, et de la même façon, hors média, les récits des proches: ce qu'ont vécu et raconté famille et voisinage. Mais dans la multiplication et la diversification des sources se retrouvent la convergence des informations et leur utilisation pour l'élaboration de scénarios cohérents.

Certains exemples sont à cet égard très instructifs.

Ainsi, pour décrire - et déplorer - les camps de concentration, Andrée, la couturière déjà citée, utilise et articule trois sources (entretien $n^{\circ} 303$ ). La première est son expérience propre: habitant près du camp de Drancy, elle a pu, des environs, en observer fonctionnement et évolution. Seconde source: ce que lui a appris son entourage (son frère connaissait par exemple une famille de déportés). Troisième source enfin, ce qu'elle a vu au cinéma à la Libération (ou encore plus tard à la télévision). L'ensemble des informations issues de ces diverses sources se fond en un seul récit, dont la cohérence est manifeste.

Un autre exemple peut permettre de souligner d'autres traits de cette élaboration scénaristique: il s'agit du récit de la guerre à Marseille que propose une narratrice qui était à l'époque une jeune mère de famille (entretien $\mathrm{n}^{\circ} 216$ ). Elle aussi mêle à son expérience directe diverses autres sources: elle évoque ainsi en une même série des événements auxquels elle a assisté (un bombardement italien de la ville en 1940), d'autres qu'elle a appris par des proches (ce qu'il advint du "père du pauvre pépé " pendant le même bombardement italien) ou par la radio (le grand bombardement allié du 27 mai 1944). Mais son récit enchevêtre également des savoirs de différentes époques: elle raconte souvent des faits qu'elle a appris après les hostilités (par exemple le rôle de son mari dans la Résistance), soit peu après (par son mari), soit longtemps après (par une camarade de Résistance de son mari retrouvée dans un club du troisième âge). Il y a enfin tout ce qu'elle a appris beaucoup plus tard par les médias (ici principalement radio et livres) : le comportement des Italiens dans les casernes marseillaises, les détails de la guerre outre-mer, etc. 
Frappe une fois de plus la façon dont la narratrice puise à ces sources diverses pour construire un récit unique et met en œuvre pour cela des procédures d'échange entre sources.

D'une part, elle se réapproprie ce qu'elle a appris par les médias. Ainsi la destruction de la flotte française à Mers El-Kébir, bien que connue pour l'essentiel par des émissions de radio récentes, est intégrée dans un univers domestique où elle prend des allures de perte familiale: ce qu'ont bombardé ces coupables Anglais, c'est "notre belle flotte qu'on avait mise de côté ».

Inversement, la relation de l'événement vécu intègre l'information ultérieure qui rétroagit sur sa présentation. Voici par exemple en quels termes est raconté l'appel lancé par le général De Gaulle le 18 juin 1940: "C'était un lundi à une heure de l'après-midi que je l'ai entendu au poste sans le vouloir. Je ne sais pas pourquoi. J'ai mis le poste pour avoir des informations, et qu'est-ce que j'entends, la voix de De Gaulle que je ne connaissais pas" (entretien $n^{\circ} 216$ ).

Se superposent manifestement ici la vision d'époque (elle ne connaît pas la voix) et la vision rétrospective (comme si elle l'avait reconnue, elle dit: "Qu'est-ce que j'entends?").

\section{- FUSIONS}

La continuité des savoirs issus de sources diverses se marque parfois très formellement par la façon dont le récit passe insensiblement de l'un à l'autre. Le fait se révèle avec une particulière netteté quand la forme employée est contradictoire avec l'information donnée. Ainsi dans cette évocation de la Résistance:

Et ceci petit à perit nous a conduits à être pendus du matin au soir au pom-pom-pom de Londres / Ici Londres / les Français parlent aux Français / Londres nous tenait au courant de ce qui se passait / Et c'est là que nous avons appris que quelque temps après, un débarquement pouvait se produire / C'est là que nous avons appris qu'il s'était produit / Et c'est là que nous avons appris qu'un groupe de résistance s'était formé à Charleval [le village du narrateur] (entretien $n^{\circ} 256$ ).

Il est évident, et la suite du récit le confirme, que l'information sur le groupe de résistance locale n'est pas venue de 
Radio Londres. Mais l'unité rhétorique que réalise l'anaphore ("c'est là que») montre bien que pour le narrateur, il s'agit d'un même ensemble d'événements, d'un même pan à la fois de l'expérience et du récit.

Plus nets encore sont les exemples où s'opère un transfert des marques de l'expérience directe et des formules qui insistent sur son authenticité ("j'ai vu») aux autres sources de savoir ou d'images. Ainsi dans cette phrase étonnante qu'emploie une narratrice pour introduire le récit d'une scène à laquelle elle n'a manifestement pas assisté: "J'ai vu mon mari aller dans un cafél et puis sur la table du café / il y avait des cadavres allongés d'une maison d'à côté" (entretien n 378). Le même "j'ai vu" normalement employé pour cautionner le témoignage direct cautionne ici le témoignage d'autrui. Pareillement, les "j’ai vu au cinéma» deviennent très souvent des "j'ai vu" tout court.

Le processus de fusion entre matériaux divers trouve son aboutissement dans des situations où l'écran et le vécu sont intégrés par le scénario dans un même espace-temps.

Ainsi à propos de la défense antiaérienne de la ville du Havre:

Hope and glory / là / j’ai été le voir / c'était sur Londres/ [...] Question de bombardements / Le Havre était entouré de saucisses / c'était un genre de ballons [...]/ on les voit très bien dans ce film-là / et c'était une matière, un peu en soie, intérieur caoutchouté / c'était gris clair [...] Moi, j’ai fait des imperméables à mes enfants là-dedans / des pèlerines avec la capuche / et puis ils ont même été à l'école avec (entretien n" 378).

Qui s'attendrait à ce que les ballons vus à l'écran dans un film de fiction (Hope and Glory, John Boorman, 1987) - reconstitution historique - puissent être utilisés par la narratrice pour confectionner des vêtements?

Dans cette construction d'un espace-temps unique qui emprunte à la fois au cinéma et au vécu, on retrouve la fusion déjà constatée dans l'exemple introductif de Jeux interdits. On retrouve aussi un mode de composition souvent employé par le cinéma lui-même, notamment dans les films historiques, lorsqu'ils mêlent aux images de fiction des images de documentaires ou d'actualités. Citons, pour rester dans les mêmes thématiques, 
l'exemple d'Allemagne mère blafarde (Helma Sanders-Brahms, 1980) : la scène de l'accouchement dans Berlin bombardé fait alterner des plans de l'héroïne sur son lit d'hôpital (élaborés pour la fiction) et des plans d'avions et de bombes repris d'actualités de l'époque.

On peut noter au passage que la répartition classique entre fiction et documents qu'opère le film d'Helma Sanders-Brahms ne se retrouve pas dans les récits de vie. Alors que les films empruntent en général des images documentaires (qui leur fournissent décor et contexte), les récits de vie empruntent toutes sortes d'images et font apparemment le même usage du document (actualités, reportages) et de la fiction (la reconstitution historique). Ainsi, lorsqu'on peut repérer que les films sur lesquels s'appuient les scénarios du vécu sont des films de fiction (que les titres en soient précisés ou que l'indiquent de façon générique le contexte et les termes employés - "le cinéma", "les films»), le plus frappant est que la question de leur statut n'est pratiquement jamais soulevée. En utilisant ainsi indifféremment des images de l'Histoire pourtant élaborées différemment, les récits de vie prennent acte d'une confusion de plus en plus fortement attestée dans notre culture contemporaine: on sait par exemple que les spécialistes se déclarent aujourd'hui incapables de distinguer parmi les images de la bataille de Stalingrad les documents authentiques et les reconstitutions ultérieures ${ }^{4}$. Il n'est pas surprenant qu'il en aille de même pour nos témoins.

\section{- SÉLECTIONS}

Dans le dialogue entre les récits de vie d'une part, les récits du cinéma et du monde en général d'autre part, nous avons insisté jusqu'ici sur la façon dont les scénarios du vécu intègrent les apports extérieurs dans leur logique propre et mettent en œuvre pour cela diverses procédures d'appropriation. La construction de cette cohérence narrative a un autre versant, du côté de la sélection des matériaux empruntés.

On constate en effet de façon générale que l'emprunt au cinéma, comme aux autres sources, n'a rien d'automatique. Il ne se produit que dans la mesure où le matériau emprunté peut 
s'intégrer dans la cohérence du récit: pour que les témoins reprennent l'Histoire que d'autres voix leur ont racontée, il faut qu'ils puissent l'intégrer dans leur Histoire à eux, faite à la fois de leur propre vécu et de la mémoire qu'ils s'en sont construite. Aussi, la convergence constatée entre les apports des diverses sources n'a-t-elle rien d'un hasard; car s'il n'y a pas continuité, il risque fort d'y avoir rejet : loin de toute ingérence inévitable, qui permettrait aux médias — ou à autrui - d'imposer leur récit, le scénario du vécu accepte seulement ce qui fait sens dans sa logique et il récuse ce qui la contredit.

L'exemple des images de camps de concentration diffusées après la Libération est à cet égard significatif. Ces images ont été massivement accueillies par les narrateurs français qui les citent et les commentent abondamment. A l'inverse, une narratrice sarroise raconte comment, à la fin de la guerre, ses voisins allemands quittèrent une projection de films sur les camps en protestant qu'il ne pouvait s'agir que de propagande américaine (entretien $\mathrm{n}^{\circ} 318$ ). De même, sur la collaboration ou sur tel épisode de la Résistance, les témoignages extérieurs sont plus d'une fois récusés, d'où qu'ils viennent (film, télévision, livre), dès lors qu'ils contredisent ce que le narrateur connaît ou pense (entretiens $\mathrm{n}^{\mathrm{os}} 175,238$ et 256 ).

De façon comparable, il peut arriver que le récit de vie s'emploie à refuser les hiérarchies établies par le récit médiatique. Une narratrice s'insurge par exemple en ces termes contre les choix de l'écran: "La libération de Paris / d'accord / mais enfin / bof / moi qui ai connu autre chose / quand j'ai vu qu'ils ne nous donnaient pas en actualités / j'ai dit bof / il s'est passé des choses / c'est certain / puis il y a des choses qu'on n'a pas sues non plus" (entretien $n^{\circ} 378$ ). Elle-même choisira au contraire d'insister sur l'importance et l'éclat de la libération du Havre.

\section{Fonctions}

L'ensemble des exemples et des analyses présentés jusqu'ici permet de dégager clairement un premier ensemble de conclusions. Tout d'abord, les scénarios du vécu participent pleinement du phénomène général de l'«intertextualité»; en dépit de 
leur caractère personnel et de leur rapport avec l'expérience directe, ils se construisent dans un dialogue intense avec les "récits du monde» qui les nourrissent et les complètent de multiples façons. Mais cette relation n'a rien pour autant d'une dépendance ou d'un asservissement: au contraire, l'analyse fait apparaître avec un relief souvent saisissant comment l'emploi de procédures de sélection et d'appropriation convergentes permet d'élaborer des scénarios de vie qui intègrent dans leurs cohérences propres les matériaux hétérogènes qu'ils utilisent.

Il est cependant une autre façon d'interroger le dialogue ainsi mis en valeur: au-delà du constat des échanges et des imbrications, on peut se demander non plus seulement comment, mais pourquoi les récits de vie recourent à cette matière extérieure. Il est apparu clairement que la matière empruntée complète les récits. Mais quelle est la spécificité de ce complément? Quelles fonctions remplit-il? A quels besoins vient-il répondre? Quelles lacunes vient-il combler? Pour répondre à ces questions, la richesse des exemples filmiques va nous conduire à recentrer principalement notre analyse sur le cinéma.

\section{- PARTAGE}

Premier élément de réponse: le film, et plus largement le récit médiatique, a sur l'expérience personnelle cet avantage qu'il est déjà très largement socialisé. Aussi peut-il apporter dans le dialogue la possibilité d'une référence commune et un ensemble de savoirs partagés sur lequel appuyer la communication. De même que, dans nos entretiens, grands-parents et petits-enfants se comprennent d'autant mieux qu'ils partagent la connaissance des mêmes personnes et des mêmes lieux, de même, entre interlocuteurs de générations et d'horizons différents, le cinéma fournit une sorte de passé commun, de paysage familier. On ne raconte pas à partir de zéro. Il ne s'agit que de poursuivre un récit que le film a déjà commencé :

La Résistance fer / surtout les cheminots / ils ont agi / il y a un film qui fait bien voir le train qui n'arrive jamais / La Bataille du rail (entretien n" 317 ).

Terrible ça a été les rafles de Juifs / tu as dû voir des films comme ça (entretien n"223). 
C'étaient les bagarres dans les rues / les Américains / tu voyais la Military Police / tu n'as jamais vu des films comme ça: quand ils arrivent avec la jeep (entretien $n^{\prime \prime 223) . ~}$

Dans ces exemples et dans bien d'autres (voir les références nombreuses et très parlantes - à des émissions de télévision récentes), la logique est toujours la même. La référence est une façon de dire: «tu peux reconnaître ce dont je te parle, puisque tu le connais déjà."

\section{- AUTORITÉ}

Autre raison de recourir au film : il apporte au récit de vie son prestige et son autorité. Il vient consacrer la valeur et l'intérêt des faits évoqués: ce que je dis vaut la peine d'être raconté puisque c'est ce qu'on voit au cinéma ( Il n'y a pas que dans les films que c'est vrai", entretien $n^{0} 378$ ). "Il m’est arrivé de manger debout devant une cuisine roulante sous la pluie / Ah oui / Debout sous la pluie / comme on voit dans les films d'ailleurs" (entretien n ${ }^{\circ} 209$ ).

Flagrante dans des cas où la référence cinématographique surgit ainsi comme moyen d'une soudaine reconnaissance, la consécration que le vécu trouve ici dans sa présence à l'écran peut aussi venir - en amont - de sa rencontre avec la caméra. "Cette année-là / on a été filmé et passé aux actualités Paramount» (entretien $\mathbf{n}^{\circ} 378$ ) : l'ex-midinette qui conclut ainsi son évocation de la célèbre fête des Catherinettes ${ }^{5}$ ne saurait mieux marquer l'intérêt de son récit. Rien de très rare dans ce constat: la fonction de la référence au cinéma est ici celle que remplit dans le commerce ordinaire de nos sociétés la mention "vu à la télé», qui souligne quotidiennement la valeur ajoutée aux événements, personnes ou objets par leur passage à l'écran. Ce recours au prestige des médias prend parfois pour s'exprimer des formes aussi directes que naïves: "Tout ce que je te dis / je l'ai lu dans des livres qui ont de la valeur» (entretien n"216).

\section{- RENFORCEMENT}

Cependant, ce que manifestent pareillement ces deux phénomènes complémentaires (le film apporte complicité dans la 
narration et consécration au récit), c'est qu'il est nécessaire, même pour raconter sa propre histoire, de retrouver une Histoire déjà racontée. Ce besoin d'appui s'est traduit parfois très concrètement: au début des entretiens, certains narrateurs sortaient des dossiers où ils avaient conservé des coupures de presse. Joue plus subtilement le même rôle l'intégration dans le récit d'éléments emblématiques, qui signalent la conformité du récit produit à un scénario attendu, à la fois reconnu et reconnaissable.

Exemple significatif: l'histoire de Marseille dans les années trente. Elle a été marquée par deux événements retentissants: l'incendie des Nouvelles Galeries (un grand magasin du centre ville) qui fit des dizaines de victimes et eut des répercussions politiques importantes et durables, et l'assassinat du roi de Yougoslavie, tué par un opposant oustachi lors d'une visite officielle. Parmi les narrateurs marseillais, rares sont ceux que le hasard a conduits à assister personnellement à ces événements exceptionnels; mais plus rares encore sans doute sont ceux qui ne les racontent pas (souvent du reste, ils les relatent comme s'ils les avaient vus de leurs yeux). C'est que, motifs obligés de l'Histoire de Marseille, ces faits apportent au récit à la fois leur éclat et leur caution: une caution d'autant plus nécessaire que nos témoins, qui sont comme nous l'avons dit des témoins "ordinaires", se sentent rarement autorisés a priori à raconter «l'Histoire avec sa grande hache". Ils s'assurent cette qualification en s'appuyant sur l'autorité d'une autre parole et en conformant leurs scénarios à des scénarios consacrés, dont ils intègrent à leurs récits propres les images et les mots.

Les fragments de récits qui font ainsi l'objet d'une appropriation qualifiante sont de nature diverse: on constate une fois de plus des procédures analogues mettant en jeu des matériaux différents. Les références à des événements spectaculaires ou à des images emblématiques sont fréquentes: ainsi la mention des jarrets des chevaux coupés par les rasoirs des manifestants de février 1934 ou l'image des rescapés squelettiques à l'ouverture des camps ${ }^{6}$. Mais scènes mythiques et stéréotypes langagiers interviennent aussi, avec la même fonction: on évoque ces ouvriers qui, jusqu'aux congés payés de 1936, "[...] n'avaient jamais vu la mer" (témoignage marseillais!), ou ces armées alle- 
mandes qui «[...] graissaient leurs canons avec du beurre», on jure avoir vu le roi d'Espagne Alphonse XIII " [...] allumer son cigare avec un billet de mille francs" (entretiens n" 213 et 238). Pareillement, la citation du film relayant le cliché historique, un ancien déporté reprend dans son récit les termes exacts du commentaire de Nuit et brouillard d'Alain Resnais (1955): il ne faut pas croire que "[...] l'enfer concentrationnaire n'aura été que d'un seul ternps et “d'un seul pays" "(entretien $n^{0} 238^{7}$ ). Formules brillantes ou images frappantes dont compte surtout la force qu'elles donnent à la parole ordinaire.

\section{- MODÈLES}

Ainsi donc pour raconter sa vie, il ne suffit pas de l'avoir vécue, mais il faut aussi trouver les mots pour la dire. Plus profondément encore, il faut trouver les schémas pour la construire: l'expérience ne peut devenir récit que par référence à des modèles qui permettent de l'organiser parce qu'ils fournissent une structure dramatique dans laquelle intégrer les faits et parce qu'ils offrent des rôles aux personnages. Grand pourvoyeur de scénarios depuis son invention, le cinéma, et particulièrement le cinéma de fiction, joue de ce fait un rôle central: en même temps qu'il apporte sa consécration au vécu, il apporte des emplois aux narrateurs et des logiques aux récits. Significatifs à cet égard sont les cas où, racontant leur propre histoire, les narrateurs se retrouvent à raconter explicitement des scènes de films. Ainsi quand l'ouverture de Jeux interdits se superpose au récit de l'exode, et dans tous les exemples du même type, il est clair que l'aventure racontée par le film donne sa forme à la relation du vécu et que la correspondance de la vie et de l'image permet aux protagonistes (en général les narrateurs) de devenir des héros de cinéma. De ce phénomène de projection, nous avons vu dès notre premier exemple à quel degré il pouvait parfois porter la confusion des niveaux : comme dans ces images de magazine qui invitent les adolescentes d'aujourd'hui à donner leur visage à l'héroïne du Titanic (James Cameron, 1998) et à faire entrer par ce biais leur réalité dans le mythe.

Au terme de ce processus, les récits peuvent devenir de véritables remakes. 
Remake du Cuirassé Potemkine. 1936, une usine occupée par des ouvriers en grève. Arrivent les gardes mobiles:

Moi, je montais sur les grilles / Il y avait à peu près deux cents gardes mobiles / Ils venaient sur quatre rangs / en marche comme les soldats marchent / Et alors je les avais interpellés là / à peu près à cinquante mètres / en disant que c'étaient des ouvriers / et qu'il y avait peur-être de leurs frères là / les gardes mobiles se sont arrêtés (entretien n"76).

Remake de la scène finale du Grand Jeu (Jacques Feyder, 1934). Sous l'Occupation, une jeune fille s'est liée d'amitié avec des officiers allemands, ses voisins. Au cours d'une soirée, elle fait les lignes de la main à un charmant petit lieutenant: "Il me dit / jai compris / Je lui dis / oh vous savez / tout peut se tromper même les lignes de la main / oh non il me dit / j'ai compris / Et il a été tué trois mois après" (entretien n"220).

Bien quaucune référence explicite ne soit faite ici au cinéma, les rencontres sont flagrantes. Elles montrent bien comment le film propose rôles et situations et peut servir ainsi de modèle narratif.

Cette fonction toutefois n'est pas réservée au cinéma. Citons l'analyse d'un exemple pittoresque qui emprunte logique et détails au roman d'aventures.

[Un] fils d'émigrants arméniens à Marseille parle de la guerre d'Espagne, qui éclate quand il a 14 ans:

"Ce n'étaient pas les quelques brigades internationales souvent raccolées par la force / comme au temps des dragons de Louis XIV aux siècles passés / et ensuite la petire aide russe qui pouvait faire grand-chose $[\ldots]$ un soir dans un petit bistrot une jeune femme a séduit soûlé pour ainsi dire un slave apatride lequel s'est réveillé le lendemain en haute mer embarqué malgré lui sur une goélette espagnole en partance pour Valence / et cette jeune femme a touché pour ce travail une pièce en or / cécait le tarif paraîr-il."

Évidemment, pour avoir "shangaïé»... un slave apatride sur une goélette, on ne peut être payé qu'en pièces d'or (entretien n $\left.{ }^{\circ} 189^{\circ}\right)$. 
La référence ici implicite à Stevenson (ou Traven?) est ailleurs référence à Delly et à ses romans «à l'eau de rose» ou encore à la rédaction scolaire", les références au cinéma restant cependant à la fois les plus fréquentes et les plus manifestes.

\section{- RECOURS}

Au bout de cette logique par laquelle films et romans prêtent leur forme au récit vécu, non seulement raconter le film de sa vie ne se distingue plus vraiment de raconter un autre film, mais le récit du film peut finir par tenir lieu du récit de la vie. Il permet alors de dire par procuration les souvenirs qui, inconscients ou trop douloureux, ne peuvent pas être exprimés directement: il permet de donner forme à l'indicible.

Ainsi dans l'exemple de Jeux interdits, la narratrice, quand elle raconte la mort des parents de Brigitte Fossey, ne dit rien de sa propre mère, qui l'accompagnait dans l'exode. Or la suite du récit nous apprend qu'elle-même à ce moment a, au sens propre, "perdu» sa mère, qui n'est pas morte, mais qu'elle n'a retrouvée que plus tard, après plusieurs jours sans nouvelles. La mort des parents du film figure dans le récit la mort possible de la mère réelle, mort d'autant plus difficile à dire qu'elle ne s'est pas produite, bien qu'elle ait pu être redoutée.

Le processus de déplacement est analogue, malgré des apparences différentes, dans un récit dont les références explicites au cinéma soulignent la très forte correspondance à un modèle d'épopée ouvrière. La narratrice raconte (sans s'y mettre en scène) des films soviétiques qu'elle a pu voir avant la guerre dans des circuits militants (entretien $n^{\circ}$ 236): Le Chemin de la vie (Nicolas Ekk, 1931), L'Évasion de Lénine (?) et une mystérieuse Sieste du prolétaire. Dans ces récits rapportés règnent à l'évidence les mêmes valeurs que dans le récit vécu (travail, courage, honnêteté, discipline). Et le récit vécu reprend plus d'une fois aux films leurs accents héroïques. Ainsi lors de cette manifestation où la narratrice, alors en fin de grossesse, s'insurge contre la violence des policiers: "Ils m'ont répondu grossièrement / puis alors ils venaient vers moi / Je leur ai bien pointé mon ventre devant / Ben tape si t'es pas un lâche» (entretien $n^{\circ} 236$ ). 
Seule différence du cinéma à la vie: les happy ends y arrivent plus vite. On peut alors penser que si l'évocation des films victorieux se fait avec une telle insistance, c'est précisément parce qu'ils constituent les seuls termes positifs dans le récit d'une période assez sombre. À la fois exemple et modèle, le cinéma fournit ici, comme jadis le conte, un récit mythique qui sert à fonder valeurs et morale.

\section{- LIMITES}

Dans ce processus où le récit de la vie se modèle sur l'imaginaire cinématographique, le partage entre vie et récit finit par perdre toute évidence. Et si les remakes étaient les événements eux-mêmes et pas seulement les récits qui en sont faits? Parfois, en effet, c'est à l'image que les narrateurs rencontrent pour la première fois des événements qui ensuite se (re)produisent dans leur vie. Ainsi lorsqu'une narratrice raconte comment elle a été à 70 ans expulsée de son logement des Halles à Paris: "On a montré à la télévision / Quand on montrait tout ça / on voyait les gens avec leur baluchon / on disait / ça y est / on les a fichus dehors / ils sont là dans une chambre / vous vous dites / ça ne m’arrivera jamais / Alors quand ça vous arrive / ça vous détruit» (entretien $\mathrm{n}^{\circ}$ 303).

\section{Conclusion}

L'investigation nous amène ainsi, comme annoncé, à constater la précarité des frontières entre les genres. Entre film et vie, comme déjà entre scénario de fiction et scénario du vécu, le jeu des échanges et des rencontres ne permet plus de savoir clairement quel terme est premier : est-ce l'histoire ou son récit? Mais à y réfléchir, cette hésitation se trouve déjà dans le mot "scénario" lui-même. On peut se référer aux emplois courants (par exemple journalistiques) du terme : qu'on déroule le "scénario" d'une crise passée ou qu'on esquisse le "scénario " d'une guerre à venir (fréquemment par exemple la supposée troisième guerre mondiale), le "scénario", dans le langage courant, sera tantôt récit, tantôt programme.

Cette double orientation peut fournir l'axe de notre conclusion. Notre recherche a permis de mettre en lumière, par le 
cinéma et au-delà de lui, l’importance et la généralité de la circulation des scénarios entre les diverses formes de récits et d'expériences, mais également la complexité des relations entre les divers éléments en jeu. Cependant, du fait de cette complexité, notre étude elle-même doit être considérée plus comme un programme que comme un bilan: non pas récit d'une recherche achevée, mais base pour le scénario de recherches à venir, dont l'objectif serait par exemple de préciser les déterminations qui régissent, en fonction des caractéristiques croisées des expériences vécues et des situations de paroles, dans leur dimension à la fois individuelle et collective, le choix de tel ou tel modèle de récit et la construction d'un scénario plutôt que d'un autre. Quelques analyses spécifiques, concernant par exemple la façon dont peut se dire le pouvoir, ou la comparaison entre la parole des hommes et la parole des femmes ${ }^{10}$, ont montré que des expériences de vie comparables peuvent conduire à des récits très différents suivant la position des narrateurs aussi bien dans l'événement qu'au moment du récit. Dans la même direction, les repérages partiels proposés ici autour du cinéma pourraient se poursuivre par des études qui s'attacheraient aux contenus autant qu'aux procédures. Quels scénarios les narrateurs mettent-ils en ceuvre pour quels usages? On pourrait tenter de le préciser en systématisant la confrontation entre scénarios de vies et scénarios de films, récit d'histoires et histoires de cinéma.

Université de Provence

NOTES

1 Pour plus de souplesse dans le mode de référence, les entretiens cités ont été numérotés. Voici la lisre des entretiens utilisés ici (numéro, nom du témoin, nom de l'enquêteur): (76) Clément Mille, par Anne Roche et Marie-Claude Taranger; (175) M. Roumieux, par Christine Rachet ; (189) Dimitri Dadena, par Alexandre Revithis; (209) Mme Iacono, par Hélène Iacono; (213) Gilberte Molinas, par Marie-Christine Vanni ; (216) Marie-Jeanne Mourtel, par Marie-Christine Peschard; (220) Mme Girolami, par Blandine Lathuillière; (223) Mme Pinkian, par Rose-Marie Arriaga; (236) Hélène Defour, par Claude Miglietti ; (238) M. Daguerre, par Josiane Trignan; (256) M. Coupille, par Magali Fabre; (283) Michel Ribon, par Hélène Stylianov; (303) Andrée, par Françoise Fabre; (313) Julie, par Geneviève Sasso; (318) Gisèle V., par Irène Pondjiclis; (378) Denise Olivier, par Nathalie Bonnabesse. Pour la liste complète et le détail de ces entretiens, voir Ânne Roche et Marie-Claude Taranger, 
C.elles qui niont pas écrit. Récits de femmes dans la région marseillaise. 1914-1945 (Aixen-Provence: Edisud, 1995).

2 L'enquête orale utilisée s'est déroulée de 1977 à 1992 dans le cadre de l'Université de Provence. La plupart des entretiens ont été réalisés par des étudiants qui ont interrogé des personnes de leur famille ou de leur entourage. Les témoins résidaient en général dans le Midi de la France (région d'Aix-Marseille), d'où la spécificité régionale très nette du corpus. La Seconde Guerre mondiale occupe une très grande place dans la plupart des récits, ce qui explique la part importante qu'elle occupe également dans nos exemples.

Notre corpus comporte plus de 500 entretiens enregistrés au magnétophone. Les extraits cirés dans le texte le sont à partir des transcriptions de ces enregistrements, transcriptions dans lesquelles nous nous efforçons de rester au plus près du texte oral (sans lui imposer par exemple la ponctuation de l'écrit). Voir Anne Roche et MarieClaude Taranger, Celles qui nont pas écrit. Récits de femmes dans la région marseillaise. 1914-1945 (Aix-en-Provence: Edisud, 1995).

3 La réflexion sur ce sujet a été amorcée dans un premier article: Marie-Claude Taranger, "Une mémoire de seconde main? Film, emprunt et référence dans le récit de vie", Hors-cadre, n"9 (1991), p. 41-60.

4 Voir Jcan-Noël Jeanneney et Monique Sauvage, Télévision, nouvelle mémoire. Les magazines de grand reportage (Paris: Seuil / INA, 1982, p. 14).

5 La fête des Catherinettes est régulièrement évoquée par les narratrices liées aux milieux de la couture parisienne. On fêtait ce jour-là les jeunes filles qui «coiffaient Saintc-Catherine", c'est-à-dire atteignaient l'âge de vingt-cinq ans sans êtrc mariées.

6 Sur ce point, voir Anne Roche, "Repeindre son passé", Revue des sciences humatines, n"192 (1983-84), p. $91-97$.

7 lee texte écrit par Jean Cayrol pour Nuit et brouillard se conclut de même: " $[\ldots \mid$ nous qui feignons de croire que tout cela est d'un seul temps et d'un seal pays, et qui ne pensons pas à regarder autour de nous, et qui nentendons pas quion crie sans fin" (c'est nous qui soulignons).

8 Pour l'analyse de cet exemple: Anne Roche, "Trous de mémoire" (communication pour le colloque, "La mémoire et l'oubli", Saint-Maximin, France, 1984).

9 Pour la référence au roman de rype Delly, voir notamment (sur les marges de notre enquête) Louise van der Wielen, Lise du plat pays (édité à compte d'auteur); pour la référence à la rédaction scolaire, l'entretien n" 216.

10 Voir Marie-Claude Taranger, "Pouvoir dire le pouvoir", (El poder en la sociedad, Barcelona: Antoni Bosch, 1986, p. 187-193); Anne Roche et Marie-Claude laranger, "Marseille, voix de femmes (1914-1945): une spécificité relative" (Marseillaises. Les femmes et la ville (collectif), Paris: Côté-Femmes Édition, 1993. p. 326-336).

\section{OUVRAGES CITES}

Jeannency, Jean-Noël et Monique Sauvage. Télévision, nouvelle mémoire. Les mayazines de grand reportage. Paris: Seuil / INA, 1982.

Roche, Anne. "Trous de mémoire" (communication), Colloque "La mémoire et l'oubli". Saint Maximin, France, 1984.

Roche, Anne. "Repeindre son passé ». Revue des sciences humaines, n" 192 (1983-84), p. 91-97.

Roche, Anne et Marie-Claude Taranger. Celles qui nont pas écrit. Récits de femmes dans La region natreillaise. 1914-1945. Aix-en-Provence: Edisud, 1995. 
Roche, Anne et Marie-Claude Taranger. "Marseille, voix de femmes (1914-45): une spécificité relative", Marseillaises. Les femmes et la ville (collectif). Paris: Côté Femmes Édition (1993), p. 326-336.

Taranger, Marie-Claude. "Unc mémoire de seconde main? Film, emprunt et référence dans le récit de vie". Hors-cadre, n"9(1991), p. 41-60.

Taranger, Marie-Claude. "Pouvoir dire le pouvoir", dans Mercedes Vilanova (direction), El poder en la sociedad. Barcelona: Antoni Bosch (1986), p. 187-193.

Van der Wielen, Louise. Lise du plat pays (à compte d'auteur).

Scénarios du vécu : cinéma, histoire et récit de vie 145 